\title{
Case management for blood pressure and lipid level control after minor stroke: PREVENTION randomized controlled trial
}

Finlay A. McAlister MD MSc, Sumit R. Majumdar MD MPH, Raj S. Padwal MD MSc, Miriam Fradette BScPharm, Ann Thompson BScPharm PharmD, Brian Buck MD, Naeem Dean MD, Jeffrey A. Bakal PhD, Ross Tsuyuki PharmD MSc, Steven Grover MD MPA, Ashfaq Shuaib MD

\begin{abstract}
- Abstract
Background: Optimization of systolic blood pressure and lipid levels are essential for secondary prevention after ischemic stroke, but there are substantial gaps in care, which could be addressed by nurse- or pharmacist-led care. We compared 2 types of case management (active prescribing by pharmacists or nurse-led screening and feedback to primary care physicians) in addition to usual care.
\end{abstract}

Methods: We performed a prospective randomized controlled trial involving adults with recent minor ischemic stroke or transient ischemic attack whose systolic blood pressure or lipid levels were above guideline targets. Participants in both groups had a monthly visit for 6 months with either a nurse or pharmacist. Nurses measured cardiovascular risk factors, counselled patients and faxed results to primary care physicians (active control). Pharmacists did all of the above as well as prescribed according to treatment algorithms (intervention).

$\mathrm{T}$ he risk of cardiovascular events is high for patients who survive a stroke or transient ischemic attack. ${ }^{1,2}$ Treatment of hypertension and dyslipidemia can substantially reduce this risk. ${ }^{3-7}$ However, vascular risk factors are often suboptimally managed after stroke or transient ischemic attack, even among patients admitted to hospital or seen in specialized stroke prevention clinics. ${ }^{8-10}$

Multiple barriers are responsible for the suboptimal control of risk factors, and traditional means of educating practitioners and patients have limited effectiveness. ${ }^{11}$ Although it has been suggested that "case managers" may be able to improve the management of risk factors, evidence is sparse and inconsistent between stud-
Results: Most of the 279 study participants (mean age $67.6 \mathrm{yr}$, mean systolic blood pressure $134 \mathrm{~mm} \mathrm{Hg}$, mean low-density lipoprotein [LDL] cholesterol $3.23 \mathrm{mmol} / \mathrm{L}$ ) were already receiving treatment at baseline (antihypertensives: $78.1 \%$; statins: $84.6 \%$ ), but none met guideline targets (systolic blood pressure $\leq 140 \mathrm{~mm} \mathrm{Hg}$, fasting LDL cholesterol $\leq 2.0 \mathrm{mmol} / \mathrm{L}$ ). Substantial improvements were observed in both groups after 6 months: $43.4 \%$ of participants in the pharmacist case manager group met both systolic blood pressure and LDL guideline targets compared with $30.9 \%$ in the nurse-led group (12.5\% absolute difference; number needed to treat $=8, p=0.03$ ).

Interpretation: Compared with nurse-led case management (risk factor evaluation, counselling and feedback to primary care providers), active case management by pharmacists substantially improved risk factor control at 6 months among patients who had experienced a stroke. Trial registration: ClinicalTrials.gov, no. NCT00931788

ies. ${ }^{12-16}$ The most recent Cochrane review on this topic concluded that "nurse- or pharmacist-led care may be a promising way forward ... but these interventions require further evaluation." ${ }^{16}$ Thus, we designed this trial to evaluate whether a pharmacist case manager could improve risk factors among survivors of stroke or transient ischemic attack. ${ }^{17}$ Because we have previously shown that hypertension control can be improved by monthly evaluation by nurses (with patient counselling and faxing of blood pressure measurements with guideline recommendations to primary care physicians) ${ }_{1}^{18}$ and this is an alternate method of case management implemented in many health organizations, we used this approach as the active control group for this study. Thus,
Competing interests: Raj Padwal is a site investigator for clinical trials for NovoNordisk and CVRx, and has received grant funding for a blood pressure cuff study. He has received personal fees for hypertension talks from Merck, Abbott and Servier and for advisory board service from Medtronic and Forest. Ashfaq Shuaib has received speaker bureau fees from CoAxia, Pfizer, BI,

Sanofi, Bayer, AstraZeneca, Lundbeck, D-Pharm,

BrainsGate, Tribute and Bristol Myers Squibb. He has received grant funding from Lundbeck, D-Pharm, GlaxoSmithKline, Asubio, PhotoThera, BrainsGate, WL Gore and Aga Medical. Ross Tsuyuki has received consultancy fees from Bristol Myers Squibb, AstraZeneca, PharmaSmart International, Merck and Abbott. He has received grant funding from Sanofi and AstraZeneca and serves on the data monitoring board for Boehringer Ingelheim. No other competing interests declared.

This article has been peer reviewed.

Correspondence to:

Finlay McAlister,

finlay.mcalister@ualberta.ca

CMAJ 2014. DOI:10.1503 /cmaj.140053 
our study represents a controlled comparison of 2 modes of case management: active prescribing (pharmacist-led case management) versus screening and delegating to primary care physicians (nurse-led case management).

\section{Methods}

\section{Study design}

We conducted a 6-month prospective, randomized controlled open-label trial, with blinded ascertainment of outcomes. Patients were screened and allocated 1:1 to the pharmacist-led case manager group (intervention) or to the nurse-led case management group (active control) between 2009 and 2012. The design details have been previously published. ${ }^{17}$

\section{Study participants}

We included patients older than 18 years who had an ischemic stroke or transient ischemic attack confirmed by a stroke specialist at 1 of the 3 stroke prevention clinics in Edmonton, Alberta. Patients were eligible if they had systolic blood pressure or low-density lipoprotein (LDL) cholesterol levels above guideline-recommended targets (average systolic blood pressure over 2 visits $>140 \mathrm{~mm} \mathrm{Hg}$, fasting LDL cholesterol $>2.0 \mathrm{mmol} / \mathrm{L}$, or total:high-density lipoprotein (HDL) cholesterol ratio $>4.0$ ). Patients were excluded if they had impaired cognition, severe disability, were institutionalized, had a condition that would preclude follow-up, had hypertensive urgency (systolic blood pressure $\geq 200 \mathrm{~mm} \mathrm{Hg}$ ) or had treatmentrefractory hypertension or dyslipidemia (i.e., already taking 3 medications and above target levels), or if they were participating in another trial.

All participants provided written informed consent, and the study protocol was approved by the Health Research Ethics Board at the University of Alberta.

\section{Nurse-led case management (control)}

All 3 stroke prevention clinics in Edmonton enrolled patients, and the attending neurologists provided written suggestions to primary care physicians with respect to treatment targets for vascular risk factors. As per local practice, the neurologists delegated ongoing management to the patient's primary care physician and rarely saw patients after the initial visit. All patients in this group also had monthly clinic visits for 6 months with a study nurse who provided lifestyle advice (exercise, low-salt diet, smoking cessation, medication adherence), checked the patient's blood pressure and LDL level, and faxed blood pressure measurements and a list of current medications to the patient's primary care physician after each visit.

\section{Pharmacist-led case management}

Our intervention involved active case management by 4 pharmacists who saw study participants monthly in a clinic setting for 6 months. Pharmacists performed the same tasks as the nurses in the control arm, as well as initiated or titrated antihypertensive and/or lipid-lowering therapy as appropriate (using treatment algorithms and targets consistent with current Canadian guidelines). ${ }^{19}$ The pharmacists did not undergo standardized training; however, they were all at similar stages of their career and were provided with the same patient educational materials and treatment algorithms. ${ }^{17}$

\section{Allocation}

Randomization was done at the time of screening by use of computer-generated random numbers with variable-sized blocked randomization stratified by stroke prevention clinic to preserve allocation concealment. Although patients were not blinded to their intervention status, all outcomes were collected in an independent and blinded manner by observers who were masked to baseline measurements and group assignment.

\section{Data collection and measurements}

Systolic blood pressure was ascertained at all study visits using the Bp TRU device (VSM MedTech). Six readings were performed 1 minute apart, and the last 5 readings were averaged. All laboratory measurements were independently analyzed at a central facility (DynaLIFE ${ }_{\mathrm{Dx}}$, Edmonton). Data collation, entry, quality assurance, and analysis were carried out at the EPICORE Centre at the University of Alberta.

\section{Outcomes}

Our primary outcome was prespecified as the proportion of participants at 6 months who attained optimal blood pressure and lipid control (defined as systolic blood pressure $\leq 140 \mathrm{~mm} \mathrm{Hg}$ and fasting LDL cholesterol $\leq 2.0 \mathrm{mmol} / \mathrm{L}$, based on Canadian clinical practice guidelines). ${ }^{19}$ This was an active control trial, so we expected improvements for all aspects of care in the control group because of active intervention by stroke specialists in the stroke prevention clinics or the primary care physicians in response to the monthly reminders about each participant's vascular risk factors, secular trends in vascular risk reduction, study volunteer and Hawthorne effects, and regression to the mean.

Data were collected for a variety of secondary outcomes (e.g., mortality, self-reported adherence, body mass index [BMI], smoking status); these are described in full in our methods manuscript. ${ }^{17}$ 


\section{Sample size}

Based on a survey of members of the divisions of neurology and general internal medicine at the University of Alberta, we determined that the minimal clinically important difference for the pharmacist case manager intervention to be considered useful was a $10 \%$ absolute improvement over and above any improvements in the active control group for our primary outcome. This required a sample size of $280 .{ }^{17}$

\section{Statistical analyses}

We used $\chi^{2}$ tests to compare the proportion of patients who attained optimal blood pressure and lipid control at 6 months (because the proportion at baseline was zero by design); we used $\chi^{2}$ testing for other binary secondary outcomes. To compare changes in systolic blood pressure, LDL cholesterol, and total:HDL cholesterol ratios between the intervention and control groups, we used 2-sample independent $t$ tests. We conducted multiple logistic regression for our primary outcome to adjust for study site and any clinically important $(>10 \%$ imbalance between arms) or statistically significant ( $p<0.1$ between arms) baseline differences.
Our primary analysis was intention-to-treat. Missing data at the 6 month follow-up assessment were imputed with a last-observation carried forward strategy; this approach conservatively assumes that all participants lost to follow-up had no change in their blood pressure or lipid levels after their last recorded value.

In a per-protocol analysis, we repeated the analyses but only included patients who attended at least 1 visit with a case manager. Because of the controversy related to blood pressure targets for patients with type 2 diabetes, we performed a sensitivity analysis in which we reanalyzed outcomes using an optimal systolic blood pressure goal of $130 \mathrm{~mm} \mathrm{Hg}$ or lower for patients with diabetes (instead of $\leq 140 \mathrm{~mm} \mathrm{Hg}$ ).

\section{Results}

Of 3696 patients screened for inclusion, 279 consented and were randomly assigned to a study group (Figure 1). All 279 participants were included in the intention-to-treat analysis. In the pharmacist-led group, 13 participants attended the baseline visit but no other visits because an

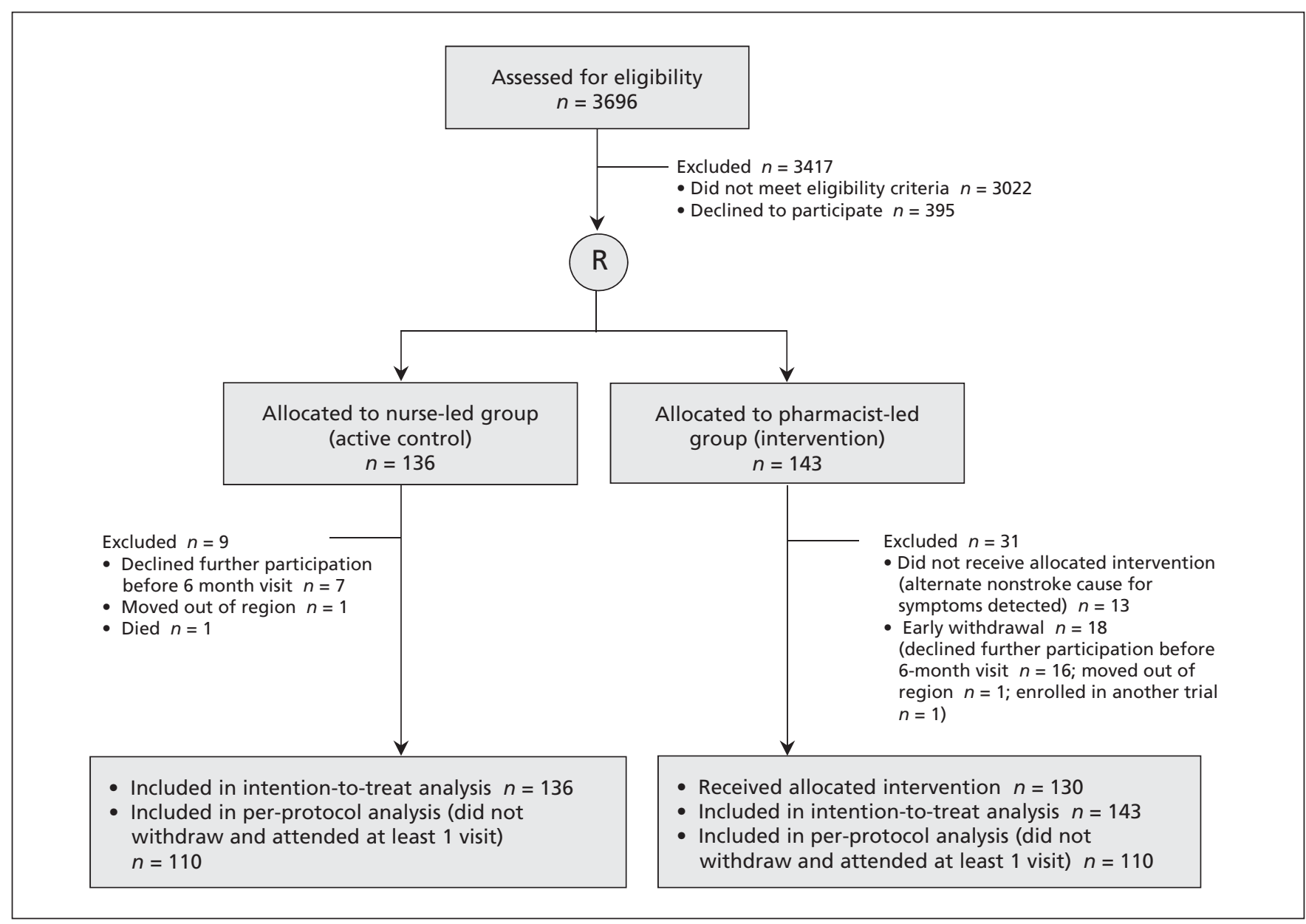

Figure 1: Flow of participants through the trial. 
alternate cause for their symptoms (e.g., brain tumour, migraine, epilepsy) was detected; these patients were included in our intention-to-treat analysis but were excluded from our per-protocol analysis because they were not exposed to any therapeutic visits with the pharmacist case manager. The baseline demographics of the 27 patients who withdrew from the study early were similar to those of the patients who did not withdraw.

\section{Baseline characteristics}

The characteristics of both study groups were similar at baseline (Table 1). The mean age was 67.6 years, $60.2 \%$ were aged 65 years or older, $58.0 \%$ were men, $15.6 \%$ had diabetes and $53.4 \%$ had a transient ischemic attack as their qualifying event. Because of our exclusion criteria, virtually all of the patients had no or only slight disability at baseline (based on modified Rankin score), but $\mathrm{ABCD}$ scores (an estimate of risk of stroke recurrence) were moderate or high in about two-thirds of patients in both arms (Table 1). Self-reported health utility and satisfaction with health care were high in both arms at baseline (Table 1). At the time of study entry, $98.2 \%$ of trial participants were taking an antiplatelet agent, $78.1 \%$ were taking an antihypertensive medication, and $84.6 \%$ were taking a statin.

\section{Optimal blood pressure and LDL control}

At baseline, none of the trial participants met both the systolic blood pressure and LDL targets recommended in the Canadian Stroke Guidelines. By 6 months, there were substantial improvements in both groups, with a clinically important and significantly greater improvement among patients in the pharmacist-led group than in the nurse-led group (pharmacist-led group: $43.4 \%$ met both systolic blood pressure and LDL goals; nurse-led group: $30.9 \%$ met both goals; $12.5 \%$ absolute difference, number needed to treat $8 ; p=0.03$ ) (Table 2). Multivariable analyses confirmed that there was greater attainment of the guideline-recommended targets in the pharmacist case manager group than in the control group (adjusted odds ratio $2.31,95 \%$ confidence interval 1.29-4.20, $p=0.005$ at 6 months; adjusted for age, comorbidities, sex, smoking status and waist circumference).

Sensitivity analysis (systolic blood pressure target of $130 \mathrm{~mm} \mathrm{Hg}$ for patients with diabetes) confirmed the robustness of our main analysis: $42.0 \%$ of patients met both systolic blood pressure and LDL targets in the pharmacist case manager group compared with $30.9 \%$ in the nurse-led control group $(p=0.04)$.

Nearly two-thirds of participants were already at systolic blood pressure targets at baseline, and patients in both groups had similar reductions in systolic blood pressure during the trial. Thus, a high proportion of participants in both groups met the systolic blood pressure target by 6 months (pharmacist-led group: 80.4\%; nurse-led group: $89.7 \% ; p=0.2$ ). The benefits of case management by pharmacists were largely driven by the higher proportion of patients who met their LDL cholesterol targets (pharmacist-led group: 51.1\%; nurse-led group: $33.8 \%, p=0.003$ ).

The per-protocol analysis (restricted to the 220 patients who did not withdraw early and who attended at least 1 study visit after the baseline visit) showed a larger treatment effect. Systolic blood pressure and lipid level targets were attained by $52.7 \%$ of patients in the pharmacist case manager group compared with $35.5 \%$ of patients in the control group at 6 months $(p<0.001)$.

The results of subgroup analyses were consistent with the main results. For example, among patients who had experienced a transient ischemic attack and who had moderate or high $\mathrm{ABCD}$ scores at baseline, control rates after 6 months were $48.7 \%$ in the pharmacist-led group compared with $26.3 \%$ in the nurse-led group $(p=0.04)$.

\section{Other outcomes}

There were no appreciable differences in the secondary outcomes unrelated to systolic blood pressure or LDL cholesterol, and there were few clinical events by 6 months ( 9 cardiovascular events and no deaths in the pharmacist-led group v. 8 cardiovascular events and 1 death in the nurseled control group) (Table 2). Patients in the pharmacist-led group had more medication changes over the course of the study (192 by the pharmacist case managers, 71 by their attending physicians, and 18 by the patients themselves) than in the nurse-led group ( 85 by the attending physician, 26 by the patients). The median number of antihypertensive medications taken at 6 months was similar in both groups (pharmacist-led group: 2 [interquartile range (IQR) 1-3]; nurseled group: 2 [IQR 1-2]; $p=0.1$ ).

At 6 months, $43.0 \%$ of patients in the pharmacist-led group were taking an angiotensinconverting-enzyme inhibitor or angiotensin receptor blocker (14\% at maximal daily dose) compared with $32.8 \%$ (5\% at maximal dose) in the nurse-led group ( $p=0.1$ for usage, $p=0.02$ for dosing). At 6 months, $58.9 \%$ of patients in the physician-led group were taking a statin $(32.7 \%$ at maximal daily dose) compared with $56.3 \%$ ( $25.8 \%$ at maximal dose) in the nurse-led group group ( $p=0.7$ for usage, $p=0.2$ for dosing).

\section{Interpretation}

Among patients with recent minor ischemic stroke or transient ischemic attack, visits with a 
pharmacist case manager with prescribing authority led to a $12.5 \%$ absolute improvement in the percentage of patients who achieved the targets for systolic blood pressure and LDL cholesterol, over and above the benefits attributable to the active control. Calling our control arm "usual care" would be a misnomer, and patients in the active control group (nurse-led group) showed a $30 \%$ absolute improve- ment in risk factor control over a 6-month period. The $43 \%$ absolute improvement at 6 months seen in our pharmacist case manager group was achieved despite the fact that over three-quarters of patients were already taking an antihypertensive or lipid-lowering medication at baseline.

Our pharmacist case manager intervention achieved greater reductions in systolic blood pres-

Table 1: Baseline characteristics of participants

\begin{tabular}{|c|c|c|c|c|c|}
\hline \multirow[b]{2}{*}{ Variable } & \multicolumn{2}{|c|}{ No. $(\%)$ or mean $\pm S D$} & \multirow[b]{2}{*}{ Variable } & \multicolumn{2}{|c|}{ No. $(\%)$ or mean $\pm S D$} \\
\hline & $\begin{array}{l}\text { Pharmacist-led } \\
\text { group } \\
n=143\end{array}$ & $\begin{array}{l}\text { Nurse-led } \\
\text { group } \\
n=136\end{array}$ & & $\begin{array}{l}\text { Pharmacist-led } \\
\text { group } \\
n=143\end{array}$ & $\begin{array}{l}\text { Nurse-led } \\
\text { group } \\
n=136\end{array}$ \\
\hline \multicolumn{3}{|l|}{ General } & \multicolumn{3}{|l|}{ Comorbidities } \\
\hline Age, yr & $68.8 \pm 11.1$ & $66.3 \pm 11.3$ & Prior stroke & $47(32.9)$ & $41(30.2)$ \\
\hline Men & $87(60.8)$ & $75(55.2)$ & Hypertension & $102(71.3)$ & $101(74.3)$ \\
\hline $\begin{array}{l}\text { Systolic blood pressure, } \\
\mathrm{mm} \mathrm{Hg}\end{array}$ & $134.9 \pm 17.53$ & $133.8 \pm 16.0$ & Coronary artery disease & $19(13.3)$ & $12(8.8)$ \\
\hline $\begin{array}{l}\text { Diastolic blood pressure, } \\
\mathrm{mm} \mathrm{Hg}\end{array}$ & $76.0 \pm 10.4$ & $77.5 \pm 10.4$ & Heart failure & $2(1.4)$ & $3(2.2)$ \\
\hline LDL cholesterol, $\mathrm{mmol} / \mathrm{L}$ & $3.20 \pm 0.84$ & $3.27 \pm 0.84$ & Atrial fibrillation & $12(8.4)$ & $12(8.8)$ \\
\hline $\begin{array}{l}\mathrm{HDL} \text { cholesterol, } \\
\mathrm{mmol} / \mathrm{L}\end{array}$ & $1.30 \pm 0.40$ & $1.27 \pm 0.38$ & Dyslipidemia & $114(79.7)$ & $116(85.3)$ \\
\hline Total:HDL ratio & $4.23 \pm 1.36$ & $4.40 \pm 1.24$ & Peripheral arterial disease & $1(0.7)$ & $2(1.5)$ \\
\hline $\begin{array}{l}\text { Glomerular filtration } \\
\text { rate, } \mathrm{mL} / \mathrm{min}\end{array}$ & $79.1 \pm 18.0$ & $79.0 \pm 21.5$ & Diabetes mellitus & $25(17.5)$ & $16(11.8)$ \\
\hline Serum creatinine, umol/L & $84.0 \pm 21.2$ & $82.6 \pm 23.3$ & Chronic kidney disease* & $17(15.6)$ & $36(29.0)$ \\
\hline Body mass index & $28.2 \pm 4.1$ & $29.5 \pm 4.9$ & COPD & $6(4.2)$ & $3(2.2)$ \\
\hline Waist circumference, cm & $95.1 \pm 11.2$ & $96.9 \pm 11.5$ & Left ventricular hypertrophy & $21(14.7)$ & $9(6.6)$ \\
\hline Men & $98.8 \pm 8.6$ & $100.3 \pm 8.7$ & Current smoker & $24(16.8)$ & $16(11.8)$ \\
\hline Women & $89.6 \pm 12.4$ & $92.6 \pm 13.1$ & Former smoker & $63(44.1)$ & $55(40.4)$ \\
\hline \multicolumn{3}{|l|}{ Qualifying event } & \multicolumn{3}{|l|}{ Medications } \\
\hline Stroke & $65(45.4)$ & $55(40.4)$ & Antihypertensive & $111(77.6)$ & $107(78.7)$ \\
\hline Transient ischemic attack & $73(51.1)$ & $76(55.9)$ & Antiplatelet & $141(98.6)$ & $133(97.8)$ \\
\hline Ocular stroke & $5(3.5)$ & $5(3.7)$ & Statin & $115(80.4)$ & $121(89.0)$ \\
\hline \multicolumn{3}{|l|}{$A B C D$ score (out of 7) } & Other lipid-lowering agent & $2(1.4)$ & $2(1.5)$ \\
\hline Low (1-3) & $20(27.4)$ & $31(40.8)$ & Warfarin & $8(5.9)$ & $6(4.7)$ \\
\hline Moderate (4-5) & $43(53.1)$ & $38(50.0)$ & \multicolumn{3}{|l|}{ Questionnaires } \\
\hline High (6-7) & $10(13.7)$ & $7(9.2)$ & $\begin{array}{l}\text { Self-reported adherence } \\
\geq 75 \% \text { for blood pressure } \\
\text { or lipid-lowering agents }\end{array}$ & $135(94.4)$ & $127(93.3)$ \\
\hline \multicolumn{3}{|l|}{ Modified Rankin score } & Overall self-rated health & $3.18 \pm 0.86$ & $3.20 \pm 0.83$ \\
\hline $\begin{array}{l}\text { No significant } \\
\text { disability }\end{array}$ & $72(85.7)$ & $70(86.4)$ & EQ-5D index score & $0.83 \pm 0.14$ & $0.86 \pm 0.12$ \\
\hline Slight disability & $11(13.1)$ & $11(13.6)$ & Health care satisfactiont & $4.28 \pm 0.75$ & $4.39 \pm 0.67$ \\
\hline Moderate disability & $1(1.2)$ & $0(0)$ & $\begin{array}{l}\text { Physical activity meets } \\
\text { Canadian guidelines }\end{array}$ & $9(6.3)$ & $6(4.4)$ \\
\hline $\begin{array}{l}\text { Note: } \text { COPD }=\text { chronic obstructiv } \\
\text { SD }=\text { standard deviation. } \\
{ }^{*} \text { Estimated glomerular filtration } \\
\text { +Scored on a 5-point Likert scal } \\
\text { fIndividuals reporting at least } 1\end{array}$ & $\begin{array}{l}\text { Almonary disease } \\
\text { e }<60 \text { based on } \\
\text { th } 5 \text { being excelle } \\
\text { ninutes of moder }\end{array}$ & $\begin{array}{l}\text { = EuroQol Healt } \\
\text { (modification } \\
\text { being poor. } \\
\text { enuous physica }\end{array}$ & $\begin{array}{l}\text { lestionnaire, } \mathrm{HDL}=\text { high-density li } \\
\text { iet in renal disease) equation. } \\
\text { ivity per week. }\end{array}$ & & \\
\hline
\end{tabular}


sure and LDL cholesterol than reported in most prior studies. ${ }^{15,20}$ Although 2 recent trials ${ }^{21,22}$ involving younger patients with uncontrolled hypertension reported similar systolic blood pressure reductions and blood pressure control rates with the involvement of pharmacist case managers, our study enrolled older higher-risk patients with lower systolic blood pressure at baseline; we also assessed the impact of a pharmacist case manager on a wider array of vascular risk factors. The observed reductions in systolic blood pressure in the nurse-led active control group were just as large as those in the pharmacist case manager group, which is consistent with our previous findings about the efficacy of our nurse-led active control intervention for blood pressure reduction. ${ }^{18}$ However, patients in the pharmacist case manager group showed much better LDL control than those in the nurse-led group. Whether this was because of greater appreciation of the importance of blood pressure control for stroke survivors and/or greater therapeutic inertia for lipid management among participating primary care physicians, or merely represents a ceiling effect for blood pressure management are points for conjecture and future study.

It is important to note that our intervention involved active medication titration by a pharmacist case manager. A study involving stroke survivors with very similar baseline features as our cohort found no benefit with case managers who did not have prescriptive authority and who merely advised patients with blood pressure measurements above target to see their primary care

Table 2: Outcomes at 6 months: intention-to-treat analysis

\begin{tabular}{|c|c|c|c|}
\hline Variable & $\begin{array}{l}\text { Pharmacist-led } \\
\text { group } \\
n=143\end{array}$ & $\begin{array}{l}\text { Nurse-led } \\
\text { group } \\
n=136\end{array}$ & $\begin{array}{c}\text { Difference } \\
\text { (intervention - control), } \\
\mathrm{SD}^{*}(95 \% \mathrm{Cl})\end{array}$ \\
\hline At guideline-recommended systolic blood pressure level at baseline & $91(63.6)$ & $85(62.5)$ & $1.1 \%(-10.8$ to 13.1$)$ \\
\hline At guideline-recommended lipid level at baseline & $8(5.6)$ & $7(5.2)$ & $0.4 \%(-5.0$ to 5.6$)$ \\
\hline Attained guideline-recommended LDL level by 6 mo & $73(51.1)$ & $46(33.8)$ & $17.2 \%(4.9$ to 29.0$)$ \\
\hline \multicolumn{4}{|l|}{ Primary outcome } \\
\hline Attained optimal systolic blood pressure and lipid level by 6 mo & $62(43.4)$ & $42(30.9)$ & $12.5 \%(0.4$ to 24.0$)$ \\
\hline \multicolumn{4}{|l|}{ Secondary outcomes } \\
\hline Systolic blood pressure at 6 mo & $126.5 \pm 17.9$ & $122.2 \pm 13.0$ & $4.3(0.6$ to 8.0$)$ \\
\hline Mortality & $0(0)$ & $1(0.7)$ & $-0.7 \%(-0.7$ to 0.6$)$ \\
\hline Vascular event $\dagger$ & $9(6.3)$ & $8(5.9)$ & $0.4 \%(-5.4$ to 6.0$)$ \\
\hline Change in HDL cholesterol & $-0.01 \pm 0.23$ & $-0.04 \pm 0.19$ & $0.03(-0.02$ to 0.08$)$ \\
\hline Change in total:HDL ratio & $-1.13 \pm 1.13$ & $-1.03 \pm 1.07$ & $-0.10(-0.36$ to 0.16$)$ \\
\hline $\begin{array}{l}\text { Self-reported adherence of } 75 \% \text { or higher for blood pressure or } \\
\text { lipid-lowering medications }\end{array}$ & $138(96.5)$ & $132(97.1)$ & $-0.6 \%(-4.3$ to 3.7$)$ \\
\hline Change in body mass index & $-0.20 \pm 1.39$ & $-0.09 \pm 3.3$ & $-0.11(-0.70$ to 0.48$)$ \\
\hline $\begin{array}{l}\text { Current smokers at baseline who stopped during } \\
6 \text {-mo period }\end{array}$ & $4 / 24(16.7)$ & $6 / 16(37.5)$ & $-20.8 \%(-46.9$ to 10.5$)$ \\
\hline
\end{tabular}


physician (mean change in systolic blood pressure $0.3 \mathrm{~mm} \mathrm{Hg}$ at 12 months). ${ }^{23}$ Indeed, several other studies have also found minimal benefit if case management only involves monitoring of risk factors, patient counselling and feedback to patients and/or primary care physicians. ${ }^{14,16,24,25}$

\section{Limitations}

Our trial was too short and was underpowered to detect changes in clinical events. However, the outcomes we evaluated (systolic blood pressure and cholesterol levels) are well-validated predictors of subsequent stroke and cardiovascular events, and there is a robust evidence base supporting blood pressure and lipid optimization for secondary prevention, making a very large outcomes-driven trial unnecessary, in our opinion. For example, lowering systolic blood pressure by $10 \mathrm{~mm} \mathrm{Hg}$ ultimately confers a $41 \%$ reduction in stroke and $22 \%$ reduction in coronary events, even among patients with vascular disease. ${ }^{26}$ In the same vein, statins are beneficial for stroke survivors, and the magnitude of benefit appears to be directly related to the degree of LDL cholesterol reduction achieved. ${ }^{4,27}$ The EXPRESS study showed (in a controlled before-after design) that prompt optimization of risk factors after transient ischemic attack resulted in an $80 \%$ reduction in recurrent stroke. ${ }^{28}$

However, there are some limitations of our trial. First, we had 6-month measurements of LDL and blood pressure for only 225 patients; however, our primary analysis was intention-totreat using the last observation carried forward, which would have biased our results toward the null because all patients had blood pressure and/or LDL cholesterol levels above target levels at baseline.

Second, our trial could not be blinded. Although this would not influence our main outcomes (which were objectively assessed using automated blood pressure machines and fasting laboratory results), this may have resulted in biased treatment estimates for subjective outcomes (e.g., satisfaction with care, quality of life).

Third, some may question whether our data are generalizable to other settings. However, our study setting closely resembles the United Kingdom National Health Service and integrated managed care organizations in the United States, and the clinical profiles of our participants were similar to recent reports of stroke survivors from other Canadian provinces, the US, the UK and Europe. . $2,10,20,24,25,28$ When considering the external validity of our results, the under-treatment of vascular risk factors is a nearly universal phenomenon and has been documented in many other settings and for other atherosclerotic conditions. ${ }^{29,30}$

\section{Conclusion}

Although primary care physicians and specialists have made great inroads in the control of cardiovascular risk factors for patients with coronary disease over the past decade,,$^{31}$ stroke prevention strategies need to extend beyond the current emphasis on antithrombotic agents and evaluation for carotid endarterectomy. We found that a nurse-led case management program based on monthly evaluation of risk factors, patient counselling and feedback to primary care physicians improved control of key risk factors for stroke (hypertension and dyslipidemia) by 6 months. However, even greater improvements were seen among patients whose care was managed by a pharmacist case manager who was empowered to initiate and titrate medications to attain guideline-recommended targets. We believe that both approaches hold great promise, not only for patients with stroke or transient ischemic attack but also for all patients with, or at high risk of, vascular disease, and our study provides much-needed information on their comparative effectiveness.

\section{References}

1. Kaplan RC, Tirschwell DL, Longstreth WT, et al. Vascular events, mortality, and preventive therapy following ischemic stroke in the elderly. Neurology 2005;65:835-42.

2. Webster F, Saposnik G, Kapral MR, et al. Organized outpatient care: stroke prevention clinic referrals are associated with reduced mortality after transient ischemic attack and ischemic stroke. Stroke 2011;42:3176-82.

3. O'Donnell MJ, Xavier D, Liu L, et al.; INTERSTROKE investigators. Risk factors for ischaemic and intracerebral haemorrhagic stroke in 22 countries (the INTERSTROKE study): a case-control study. Lancet 2010;376:112-23.

4. Straus SE, Majumdar SR, McAlister FA. New evidence for stroke prevention: scientific review. JAMA 2002;288:1388-95.

5. Hackam DG, Spence JD. Combining multiple approaches for the secondary prevention of vascular events after stroke: a quantitative modeling study. Stroke 2007;38:1881-5.

6. Zhang H, Thijs L, Staessen JA. Blood pressure lowering for primary and secondary prevention of stroke. Hypertension 2006; 48:187-95.

7. Baigent C, Keech A, Kearney PM, et al. Cholesterol Treatment Trialists Collaborators. Efficacy and safety of cholesterol-lowering treatment: prospective meta-analysis of data from 90056 participants in 14 randomised trials of statins. Lancet 2005;366:1267-78.

8. Mouradian MS, Majumdar SR, Senthilselvan A, et al. How well are hypertension, hyperlipidemia, diabetes, and smoking managed after a stroke or transient ischemic attack? Stroke 2002; 33:1656-9.

9. Joseph LN, Babikian VL, Allen NC, et al. Risk factor modification in stroke prevention. The experience of a stroke clinic. Stroke 1999; 30:16-20.

10. Roumie CL, Ofner S, Ross JS, et al. Prevalence of inadequate blood pressure control among veterans after acute ischemic stroke hospitalization: a retrospective cohort. Circ Cardiovasc Qual Outcomes 2011;4:399-407.

11. Majumdar SR, McAlister FA, Furberg CD. From publication to practice in chronic cardiovascular disease- the long and winding road. J Am Coll Cardiol 2004;43:1738-42.

12. Clark AM, Hartling L, Vandermeer B, et al. Meta-analysis: secondary prevention programs for patients with coronary artery disease. Ann Intern Med 2005;143:659-72.

13. Shojania KG, Ranji SR, McDonald KM, et al. Effects of quality improvement strategies for type 2 diabetes on glycemic control: a meta-regression analysis. JAMA 2006;296:427-40.

14. Carter BL, Rogers M, Daly J, et al. The potency of team-based care interventions for hypertension: a meta-analysis. Arch Intern Med 2009;169:1748-55. 
15. Santschi V, Chiolero A, Burnand B, et al. Impact of pharmacist care in the management of cardiovascular disease risk factors: a systematic review and meta-analysis of randomized trials. Arch Intern Med 2011;171:1441-53.

16. Glynn LG, Murphy AW, Smith SM, et al. Interventions used to improve control of blood pressure in patients with hypertension. Cochrane Database Syst Rev 2010;(3):CD005182.

17. McAlister FA, Majumdar SR, Padwal RS, et al. The preventing recurrent vascular events and neurological worsening through intensive organized case-management (PREVENTION) Trial protocol [clinicaltrials.gov identifier: NCT00931788]. Implement Sci 2010;5:27.

18. McLean DL, McAlister FA, Johnson JA, et al. A randomized tria of the effect of community pharmacist and nurse care on improving blood pressure management in patients with diabetes mellitus: study of cardiovascular risk intervention by pharmacists-hypertension (SCRIP-HTN). Arch Intern Med 2008;168:2355-61.

19. Lindsay P, Bayley M, McDonald A, et al. Toward a more effective approach to stroke: Canadian Best Practice Recommendations for Stroke Care. CMAJ 2008;178:1418-25.

20. Vernooij JW, Kaasjager HA, van der Graaf Y; SMART Study Group. Internet based vascular risk factor management for patients with clinically manifest vascular disease: randomised controlled trial. BMJ 2012;344:e3750.

21. Margolis KL, Asche SE, Bergdall AR, et al. Effect of home blood pressure telemonitoring and pharmacist management on blood pressure control: a cluster randomized clinical trial. JAMA 2013;310:46-56.

22. Magid DJ, Olson KL, Billups SJ, et al. A pharmacist-led, American Heart Association Heart360 Web-enabled home blood pressure monitoring program. Circ Cardiovasc Qual Outcomes 2013; 6:157-63.

23. Kerry SM, Markus HS, Khong TK, et al. Home blood pressure monitoring with nurse-led telephone support among patients with hypertension and a history of stroke: a community-based randomized controlled trial. CMAJ 2013;185:23-31.

24. Wolfe CD, Redfern J, Rudd AG, et al. Cluster randomized controlled trial of a patient and general practitioner intervention to improve the management of multiple risk factors after stroke: stop stroke. Stroke 2010;41:2470-6.

25. Ellis G, Rodger J, McAlpine C, et al. Impact of stroke nurse specialist input on risk factor modification: a randomized controlled trial. Age Ageing 2005;34:389-92.

26. Law MR, Morris JK, Wald NJ. Use of blood pressure lowering drugs in the prevention of cardiovascular disease: meta-analysis of 147 randomised trials in the context of expectations from prospective epidemiological studies. BMJ 2009;338:b1665.

27. Amarenco P, Goldstein LB, Szarek M. Effects of intense lowdensity lipoprotein cholesterol reduction in patients with stroke or transient ischemic attack: the Stroke Prevention by Aggressive Reduction in Cholesterol Levels (SPARCL) trial. Stroke 2007; 38:3198-204.

28. Rothwell PM, Giles MF, Chandratheva A, et al. Effect of urgent treatment of transient ischaemic attack and minor stroke on early recurrent stroke (EXPRESS study): a prospective population-based sequential comparison [published erratum in Lancet 2008;371:386]. Lancet 2007;370:1432-42.

29. Brown LC, Johnson JA, Majumdar SR, et al. Evidence of suboptimal management of cardiovascular risk in patients with type 2 diabetes mellitus and symptomatic atherosclerosis. CMAJ 2004; 171:1189-92.

30. Bhatt DL, Steg PG, Ohman EM, et al. International prevalence, recognition, and treatment of cardiovascular risk factors in outpatients with atherothrombosis. JAMA 2006;295:180-9.

31. McAlister FA, Fradette M, Majumdar SR, et al. The Enhancing Secondary Prevention in Coronary Artery Disease trial. CMAJ 2009; 181:897-904.

Affiliations: Division of General Internal Medicine (McAlister, Majumdar, Padwal); Patient Health Outcomes Research and Clinical Effectiveness Unit (McAlister, Bakal); Epidemiology Coordinating and Research (EPICORE) Centre (McAlister, Fradette, Tsuyuki); Faculty of Pharmacy and Pharmaceutical Sciences (Thompson); Division of Neurology (Buck, Dean, Shuaib), University of Alberta; Division of General Internal Medicine (Dean), Royal Alexandra Hospital, Edmonton, Alta.; McGill Cardiovascular Health Improvement Program (Grover), Division of General Internal Medicine, McGill University, Montréal, Que.

Contributors: Finlay McAlister, Sumit Majumdar and Ashfaq Shuaib conceived the study. Finlay McAlister and Sumit Majumdar designed the study with input from all authors. All authors were involved in seeing study patients and/or collecting data. Jeffrey Bakal conducted the analyses. Finlay McAlister drafted the manuscript, and all authors provided comments on the drafts and have read and approved the final version submitted for publication. Finlay McAlister is guarantor for this work.

Funding: Finlay McAlister and Sumit Majumdar receive salary support awards from Alberta Innovates Health Solutions. Finlay McAlister holds the University of Alberta Chair in Cardiovascular Outcomes Research. Sumit Majumdar holds the Patient Health Management Chair at the University of Alberta.

Project-specific funding for this trial was provided by the Heart and Stroke Foundation of Alberta, the Alberta Heritage Foundation for Medical Research, and Knowledge Translation Canada.

None of the funders had a role in the design of the study nor in the conduct, analysis, interpretation or reporting of the study, nor access to the data.

Acknowledgements: The authors thank Bonnie Woloschuk, Debbie Boyko, Debbie Bellerose, Michelle Lovlin, Shannon Butler-Tyler, Joanna Wi (study nurses); Kirsten George-Phillips and Mylien Nguyen (study pharmacists), and the neurology staff of the participating stroke prevention clinics (Drs. Thomas Jeerakathil, Kurshid Khan, Ken Butcher, Maher Saqqur, James Scozzafava, Muzaffar Siddiqui, Mikael Murataglu) and clinic nurses (Tobey St. Arnault, Marion Fuchs, Eden Mopas). 